\title{
Coating generation: Vaporization of particles in plasma spraying and splat formation
}

\author{
M. Vardelle, A. Vardelle, K-I Li, P. Fauchais \\ Université de Limoges, 123 Avenue A. Thomas 87000, Limoges, F \\ N. J. Themelis \\ Columbia University, New York, NY 10027, USA
}

\begin{abstract}
This paper deals with three important stages which affect the quality of plasma-sprayed coatings: particle trajectories and their time variation due to plasma jet fluctuations; particle evaporation; and splat formation and cooling which are controlled mainly by splat thickness and the quality of the bonding with the underlying surface.
\end{abstract}

\section{INTRODUCTION}

Plasma spray deposition is a versatile technology that offers a reliable cost-effective solution for many industrial problems. It allows the spraying of a wide range of materials from superalloys and refractory intermetallic compounds to ceramics. This technology encompasses also the manufacture of net shapes which can be used directly as engineered materials.

However the distinctive microstructure of plasma-sprayed coatings which consist of many layers of thin lamellas parallel to the substrate surface with possible inclusions of pores, unmelted particles and crack network can curb their use. The control of this microstructure is linked to the injection conditions of the powders, the natural fluctuations of the plasma jet in both time and space modes, and the flattening and freezing of the particles impinging on the substrate.

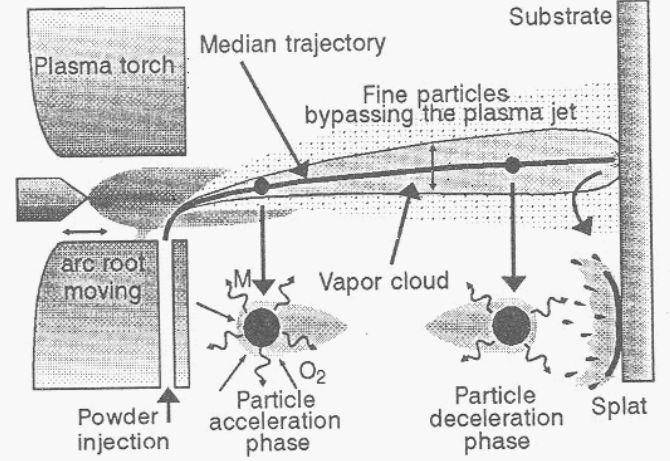

Fig. 1 Schematic illustration of particle treatment in plasma spray process.
This paper discusses the basic phenomena which affect coating formation and may explain the inhomogeneity of its morphology as well as the deposition efficiency. The first part refers to the conditions of injection of the powder and the resulting distribution of trajectories which are linked to the plasma jet fluctuations. The second part examines the behavior of particles in flight and specially their evaporation. The last part relates the interactions between particles and the underlying layer. Examples of experimental results achieved at this laboratory, illustrate the various sections.

\section{INJECTION OF THE POWDER AND THE RESULTING DISTRIBUTION OF TRAJECTORIES IN THE PLASMA JET}

In plasma spraying, the powder is commonly injected close to the nozzle exit. The momentum of a particle and the plasma jet fluctuations at the time of injection, condition the trajectory of the particle in the plasma jet and therefore its treatment.

Plasma jet fluctuations originate in the continual change of location of the arc root attachment point at a frequency ranging between 12 and $20 \mathrm{kHz}$ for commercial plasma spray torches. The arc root "restrike" mode at the anode wall gives rise to plasma puffs so that the plasma flow is a heterogeneous 
medium made up of successive puffs of hot gas surrounded by colder flow (1). This results in voltage fluctuations (up to $30 \%$ ) and variation of the rate of electric power dissipation in the plasma jet.

Moreover, when the plasma jet exits the nozzle, it expands by entraining the surrounding fluid. This entrainment process is mainly governed by the large density and velocity differences between the plasma flow and the ambient atmosphere. Large-scale eddies resulting in the engulfment of cold gas bubbles extend from the outer edge of the shear layer toward the centerline of the jet in which they are broken down into smaller eddies and mixed with the flow when they are sufficiently heated (2). In case of air environment, this may result in particle oxidation.

In the first few millimeters after the injection point, particles of 5 and $70 \mu \mathrm{m}$ go through 1 to 5 plasma puffs respectively. Therefore they may experience a different environment and their trajectories may fluctuate. This phenomenon is more marked for the smaller particles. Further downstream in the plasma jet, the trajectory of the large particles is generally unaffected by the turbulence of the plasma flow while smaller, less massive particles follow effectively the fluid motion and may be entrained in an eddy. However, heating and acceleration of the large particles depend on the successive hot and colder gas zones they traverse (3). This contributes to the rather large distribution of particle velocity and temperature which can be observed at a given point of the plasma jet or upon impact on the substrate.
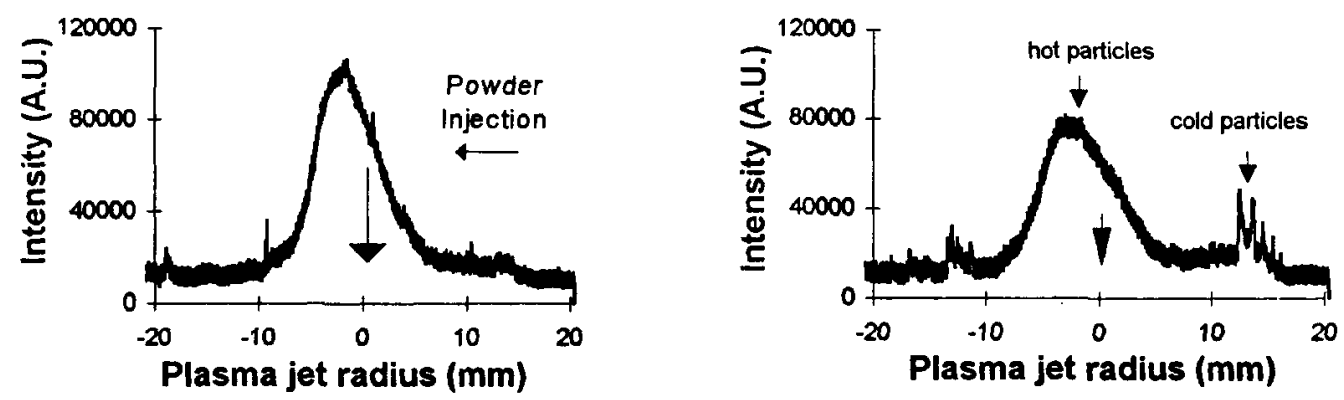

Fig. 2 Radial profiles of particle distributions randomly acquired over 13-ms intervals.

The injection and the trajectories of particles in a DC plasma jet were investigated at this laboratory using a 2-D imaging technique. The measuring system consisted of a planar laser sheet generated by an oscillating mirror and a linear diode array detection system (4). Figure 2 shows examples of light signal emitted and scattered by zirconia particles $(5-45 \mu \mathrm{m})$ as they crossed the laser sheet. These signals were randomly acquired over $13-\mathrm{ms}$ intervals at a distance of $45 \mathrm{~mm}$ from the injection point. The differences between both signals may be attributed to the natural fluctuations of the plasma jet but also to the irregularity of the powder feed rate. The two smaller peaks which occur at a radius of about 12 $\mathrm{mm}$ from the jet centerline arise mainly from small particles which, at the injection point, do not have enough momentum to penetrate the plasma jet and are therefore carried around the jet envelope. Computations and experiments (4) have indeed shown that inside the injection tube the finer particles have a tendency to tilt toward the injector wall due to turbulent dispersion. This results in a low velocity at the injector exit. These cold particles may be entrained by the gas flow further downstream and thus become embedded in the coating where they may create defects.

Measurements of the spatial distribution of particle flux density and particle velocity and temperature were obtained by using a system consisting of a high-speed two-color pyrometer and a laser doppler velocimeter (5). They show that powders have a tendency to segregate in the plasma jet according to their size, as illustrated in Fig. 3.

However, the segregation is much less than that predicted by models which do not account for distributions of injection velocity (6). The median trajectory, that is the trajectory followed by the highest number of particles is at an angle of about $3-5^{\circ}$ with the jet centerline. The widest angle is, of course, observed for the trajectories of the largest particles. Generally these particles reach a lower temperature than the smaller particles which have a lower thermal inertia and travel closer to the jet centerline and thus experience higher gas temperature. 


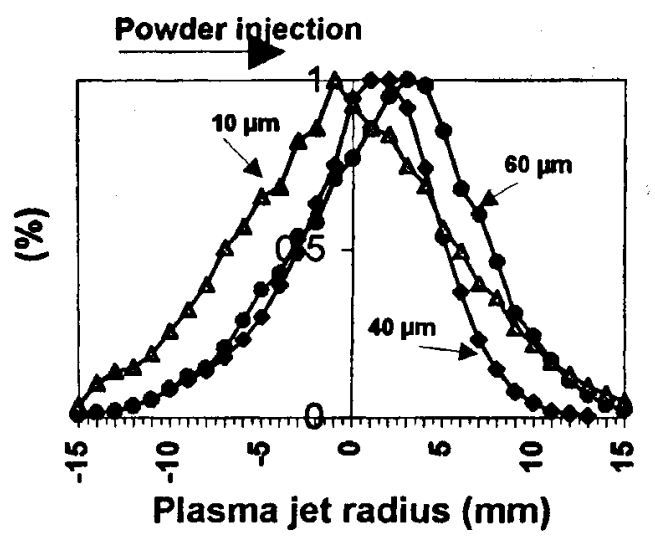

Fig. 3 Radial distribution of alumina particles for three particle sizes at $80 \mathrm{~mm}$ from the nozzle exit.

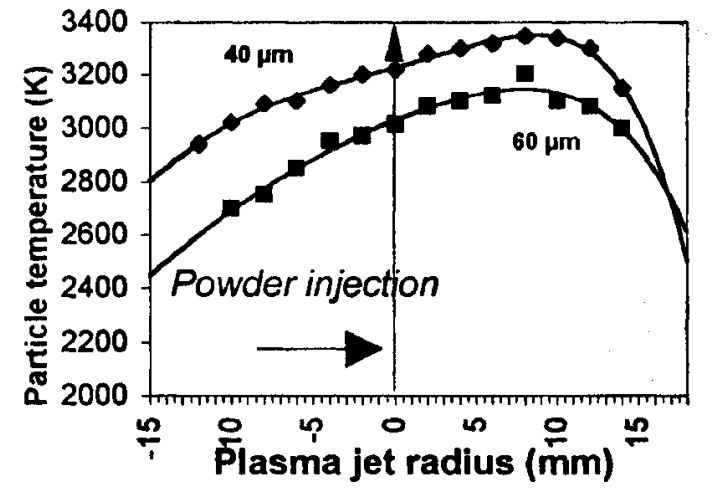

Fig. 4 Radial distribution of temperature of alumina particles for two particle sizes at $80 \mathrm{~mm}$ from the nozzle exit.

\section{EVAPORATION OF PARTICLES IN FLIGHT}

The production of dense high-strength coatings requires that the particles traverse the hottest region in the plasma jet to complete the melting process and reach high velocity. The relatively broad range of particle sizes, and the dispersion of particle trajectories in the plasma jet result in various particle timetemperature histories; the following stages take place: heating of the solid phase, melting, heating of the liquid phase and partial or total evaporation. These steps can be considered as sequential for materials with high thermal conductivity $(k>60 \mathrm{~W} / \mathrm{m} . \mathrm{K})$ such as metals and alloys. The rate of heat transfer through the boundary layer is proportional to the surface area of the particle while the amount of material to be heated is proportional to its volume. Therefore the duration of each of these four stages is proportional to the particle diameter.

TABLE 1. Rough computation for various materials, of the heat $\mathbf{Q}$ necessary for reaching the boiling temperature for 30- $\mu \mathrm{m}$ particles; and their residence time as measured by a laser velocimeter, under comınon spraying conditions $\left(\mathrm{Ar}-\mathrm{H}_{2}: 20 \mathrm{vol} \%\right.$ gas mixture, arc current: $600 \mathrm{~A}$, substrate standoff distance: $100 \mathrm{~mm}$, nozzle i.d.: $7 \mathrm{~mm}$ )

\begin{tabular}{|c|ccccccc|}
\hline Material & $\mathrm{Cu}$ & $\mathrm{Fe}$ & $\mathrm{Mo}$ & $\mathrm{Co}$ & $\mathrm{W}$ & $\mathrm{ZrO}_{2}$ & $\mathrm{Al}_{2} \mathrm{O}_{3}$ \\
\hline $\mathrm{Q}(\mathrm{J})$ & $1.4810^{-4}$ & $1.9710^{-4}$ & $1.9710^{-4}$ & $2.210^{-4}$ & $3.1410^{-4}$ & $2.6910^{-4}$ & $3.0610^{-4}$ \\
\hline $\begin{array}{l}\text { Residence } \\
\text { time (ms) }\end{array}$ & - & 1.1 & - & - & 2 & 1 & 0.8 \\
\hline
\end{tabular}

As the working conditions of the plasma torch are generally set for the average size of the particle size range, the smaller particles, which have penetrated in the flow, may evaporate after a few millimeters of travelling. The mixing of the vapor with the plasma gas can alter the heating of other particles and the evaporation lowers the deposition efficiency.

The study of particle vaporization by emision spectroscopy (7) is on line with the values shown in Table 1. It shows that the evaporation of alumina and tungsten particles is lower than that of iron particles except for fine particles $(<10 \mu \mathrm{m})$.

A simplified model of the spray process has been developed to predict the total mass fraction of powder vaporized under usual spray conditions. This model takes into account the distribution of particle size and their time-temperature history according to their size: particle temperature and residence time have been determined from experimental results obtained with a laser doppler velocimeter connected to a high-speed pyrometer (5).

The maximum rate of vaporization per unit area of a liquid surface was calculated from the Langmuir equation which predicts the maximum rate of vaporization under vacuum (8):

$\mathrm{N}_{\max }=\frac{\mathrm{P}}{\sqrt{2 \pi \mathrm{RTM}}} \quad$ where $\mathrm{P}$ is the vapor pressure of material at absolute temperature $T, M$ the molecular weight of material and $\mathrm{R}$ the universal gas constant. 
For a commercial iron powder of particle size ranged between 5 and $80 \mu \mathrm{m}$, it was found that the particle residence time ranged between 0.6 and $2.5 \mathrm{~ms}$. The temperature of the smaller particles was close to the boiling point $(3023 \mathrm{~K})$ whereas the temperature of the largest particles is about $2100 \mathrm{~K}$. Particles of size below $10 \mu \mathrm{m}$ and which have penetrated the plasma jet, are totally vaporized. The calculated fraction of vaporized mass is about $15 \%$ for a $30-\mu \mathrm{m}$ iron particle and $1 \%$ for a $60-\mu \mathrm{m}$ particle, as shown in Fig. 5 (curve 1).

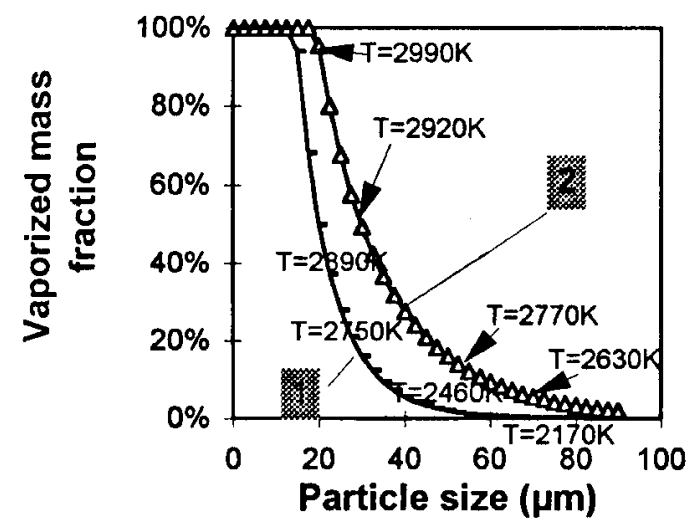

Fig. 5 Computed vaporized mass fraction and estimated particle temperature for iron particles of $5-80 \mu \mathrm{m}$

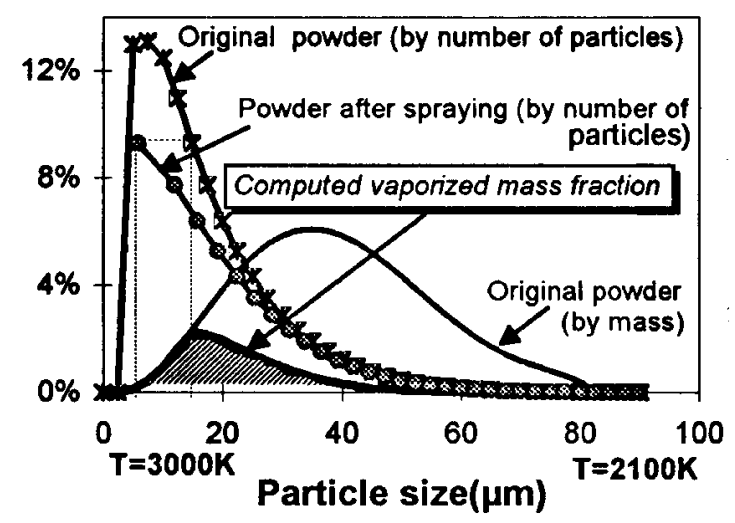

Fig. 6 Distribution of powder before and after spraying; total vaporized mass : $17 \%$.

For different spraying conditions (e.g. with an increase of arc current), the temperature of the largest particles can reach $2600 \mathrm{~K}$. In that case, the vaporization rate was about $50 \%$ for a $30-\mu \mathrm{m}$ particle and $10 \%$ for a $60-\mu \mathrm{m}$ particle (curve 2 ).

Taking into account the distribution by weight of the original powder and the time - temperature history of particles according to their size, resulted in a total mass account of vaporized material of $17 \%$. The main results of the model are summarized in Fig. 6, which shows the powder distribution before and after plasma spraying. When the temperature of the largest particles was as high as $2600 \mathrm{~K}$, the computed total amount of vaporized mass was equal to $40 \%$.

The concentration of metallic atoms produced by particle vaporization was measured by absorption spectroscopy (7). This technique made it possible to determine the atom and ion populations of ground and metatable states. The experimental setup included a hollow cathod lamp (made of the same metal as the metallic element of the vapor) which was focused into the plasma flow. The image of the focal point was then formed on the entrance slit of a monochromator. The signal was analysed by a lock-inamplifier connected with a chopper. Figure 7 shows the concentration of iron atoms at the substrate stand off distance $(100 \mathrm{~mm})$ for three particle size ranges : 14-55, 80-100 and 125-160 $\mu \mathrm{m}$ and at powder feed rate of $50 \mathrm{~g} / \mathrm{h}$.

The summation of iron atoms for particles ranging between 14 and $55 \mu \mathrm{m}$, in a plasma jet slice 1-mm thick, gives a total amount of vaporized mass of $15 \%$. This result is in fair agreement with the value predicted by the simplified model mentioned above.

Measurements show also that the presence of oxygen in the atmosphere around a metal droplet may affect its rate of vaporization. The vapor molecules must diffuse through the boundary layer surrounding the molten sphere. In an inert gas, the metal vapor pressure around the sphere builds up and the rate of vaporization decreases while in air the countercurrent molar flux of metal vapor and oxygen react and that homogeneous oxidation reaction consumes the metal vapor, thereby increasing the rate of vaporization from the droplet surface.

Figure 8 compares the observed radial profiles of the density of iron atoms produced by vaporization of iron particles injected in a argon - hydrogen plasma jet issuing either in air or argon atmosphere. The density of iron atoms is two orders of magnitude higher when the surrounding gas is air, despite a lower gas flow temperature. 


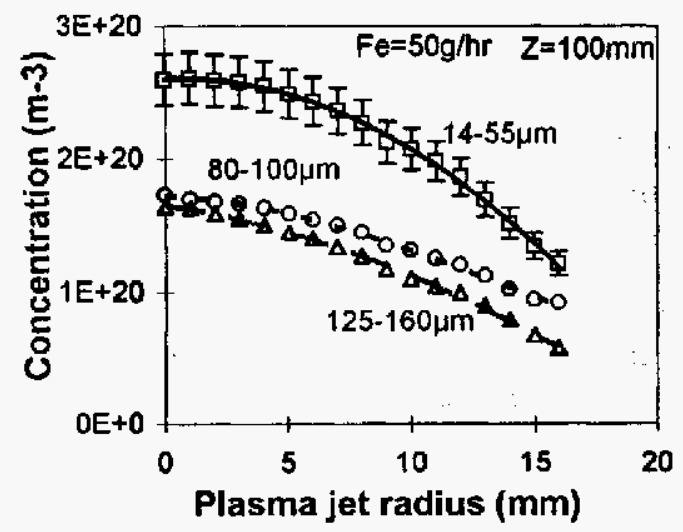

Fig.7 Concentration of iron atoms measured by absorption spectroscopy at $100 \mathrm{~mm}$ from the injection point; powder feed rate : $50 \mathrm{~g} / \mathrm{h}$.

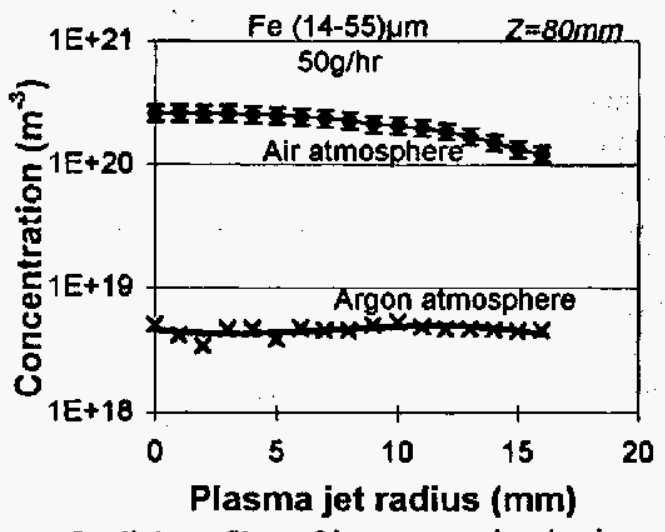

Fig. 8 Radial profiles of iron atom density in a Ar- $\mathrm{H}_{2}$ plasma jet issuing in air or argon at $80 \mathrm{~mm}$ from the injection point.

To investigate the vapor cloud surrounding a particle, a diagnostic technique based on an emission spectroscopy method has been developed at this laboratory (10). The method consistes of analysing, in both time and amplitude modes, two lines corresponding to the excited metal atoms of the vapor around a particle when that particle passes through a well-defined observation window at a known velocity. The evolution of the excitation temperature within the vapor cloud was inferred from the intensity ratio of the two lines simultaneously measured. The concentration of the metal atoms was obtained from the intensity of one line once the temperature evolution in the vapor cloud was known. Local thermodynamic equilibrium conditions were assumed.

Figure 9 shows results obtained with this method for $\mathrm{WC}+17 \%$ Co particles. The various schemas represent the evolutions of $\mathrm{Co}$ atom concentration around a particle for two axial locations from the nozzle exit ( 50 and $70 \mathrm{~mm}$ ) and at various radial distances from the jet centerline. These schemas show that the gradients of metal atom concentration are high around a particle, and that the concentration values are larger than the mean values obtained by absorption spectroscopy (see Fig. 7). The gradients are the highest at $6 \mathrm{~mm}$ from the jet centerline that is for particles which follow the median trajectory and reach the highest temperatures (see Fig. 4). Assuming that convection is low close to the particle surface, the comparison of the data achieved at 50 and $70 \mathrm{~mm}$ downstream of the nozzle exit results in a diffusion coefficient of $10 \mathrm{~cm}^{2} \mathrm{~s}^{-1}$.

The vapor cloud around a particle can also be observed at locations corresponding to substrate standoff distances. It may thus alter the flattening of particles when they impinge on the substrate: The vapor may limit the interfacial contact between the particle and the substrate and recondense in the form of very fine particles. This favors the splashing of the molten droplet on impact and the formation of pores in the coating.

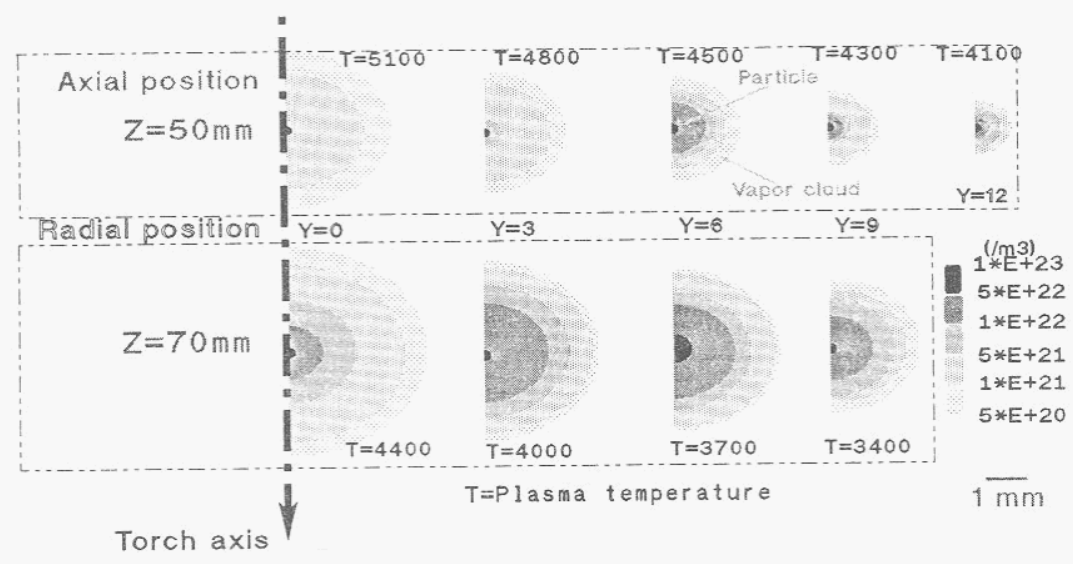

Fig. 9 Distribution of Co atoms around a particle as measured by emission spectroscopy ( $\mathrm{T}$ : bulk gas temperature, $\mathrm{K}$ ). 


\section{IMPACT AND SOLIDIFICATION OF PARTICLES}

The microstructure of the coating and thus its physical properties are mainly determined by the flattening and solidification of particles impinging on the substrate. These processes are highly related to plasma-particle interactions which condition the velocity, size and molten state of particles upon impact. They depend also on the surface topography of the substrate, its temperature and its reactivity. Additional parameters peculiar to the process such as the relative gun-to-substrate motion, and substrate time-temperature history, will also affect particle-substrate interactions.

The flattening of the droplets on the surface determines the shape and the thickness of the resulting splat, and the degree of bonding between splats or between splats and substrate. The presence of oxide layers on the droplets and/or the substrate surface, also affect the particle - underlying surface bonding. An investigation of particle impact using a high-speed pyrometer (11), has shown that the duration of the spreading of the droplet on the surface is less than $1-3 \mu \mathrm{s}$ for metal or ceramic particles. It depends mainly on the kinetic energy of the particle; the viscous effects are dominant during the spreading of the droplet while the surface tension effect, including the contact angle, could become important at the final stage deformation.

As confirmed by different numerical simulations $(12,13)$, the reduced flattening time $\tau$ ( $\tau=t$.V/d where $t$ is the flattening time, $V$ and $d$ the particle velocity and diameter respectively on impact) can be expressed in terms of a Reynolds number calculated from the particle parameters upon impact:

$$
\tau=A \cdot \operatorname{Re}^{0.2} \text { where } \mathrm{A} \text { is a constant. }
$$

Measurements have also shown that the substrate roughness has a pronounced influence on the flattening time : the latter tends to decrease with the substrate roughness which limits the flowing of the liquid parallel to the surface. The flattening degree, defined as the ratio of the splat diameter to the droplet diameter, shows also a dependence on the particle parameters on impact and on the substrate roughness (Figure 10).

There was no significant change in the duration of the flattening process when the substrate temperature was increased from room temperature up to $500 \mathrm{~K}$. However, measurements have shown that the substrate temperature and its surface topography have a drastic influence on the splat shape. Ceramic particles $\left(\mathrm{ZrO}_{2}, \mathrm{Al}_{2} \mathrm{O}_{3}\right)$ sprayed onto smooth steel substrates whose temperature was higher than $200^{\circ} \mathrm{C}$ exhibited a lenticular shape across the spray cone and had a high bonding with the substrate, provided that the latter was not oxidized. For lower substrate temperatures, splats had distorted shapes. An increase in substrate roughness was also found to increase the splashing of molten material on impact and resulted in star-shape splats with a very irregular thickness, especially for cold substrates. The quality of the contact between the splat and the substrate can be modeled by an interface thermal contact resistance. Comparisons between experimental and computed thermograms result in a resistance of about $10^{-7}-10^{-8} \mathrm{~m}^{2} \mathrm{~K} \mathrm{~W}^{-1}$ for a contact of "good" quality and $10^{-5}-10^{-6} \mathrm{~m}^{2}$ $\mathrm{K} \mathrm{W}^{-1}$ for a contact of "poor" quality.

When the solidification front in the splat advances faster than the liquid spreads on the surface, the flattening process can be limited, if not stopped. This is favored by good quality of contact between the splat and the underlying layer, and low splat thickness. Both parameters are the most important ones in conditioning the splat solidification and cooling. The cooling rate will determine the extent of undercooling, the crystal growth and the formation of metastable or amorphous phases. In plasma spraying, the estimated cooling rate over the total time of the cooling of splats is in the order of $10^{6} \mathrm{~K} / \mathrm{s}$. However, for the first microseconds following the impact, the cooling rate can be as high as $10^{8} \mathrm{~K} / \mathrm{s}$ if the splat is in intimate contact with the substrate. This high cooling rate favors the columnar growth of the crystals. Moreover the re-heating of the underlying layer by the heat flux due to splat solidification favors the epitaxial crystal growth between splats.

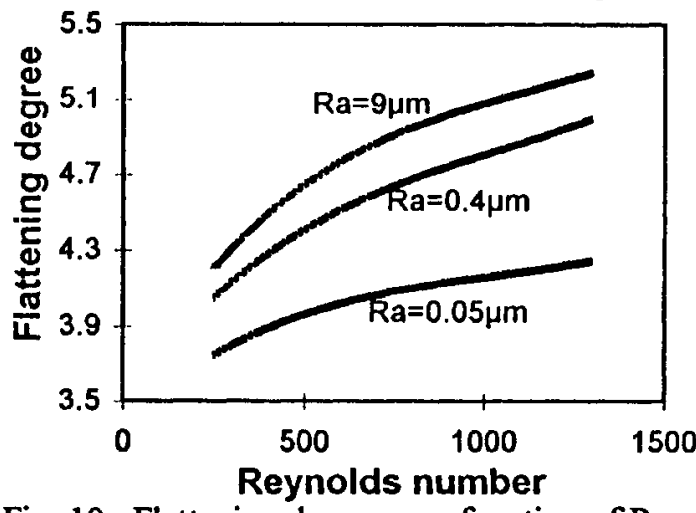

Fig. 10 Flattening degree as a function of Reynolds number at time of impact ( $\mathrm{Ra}$ : substrate roughness). 

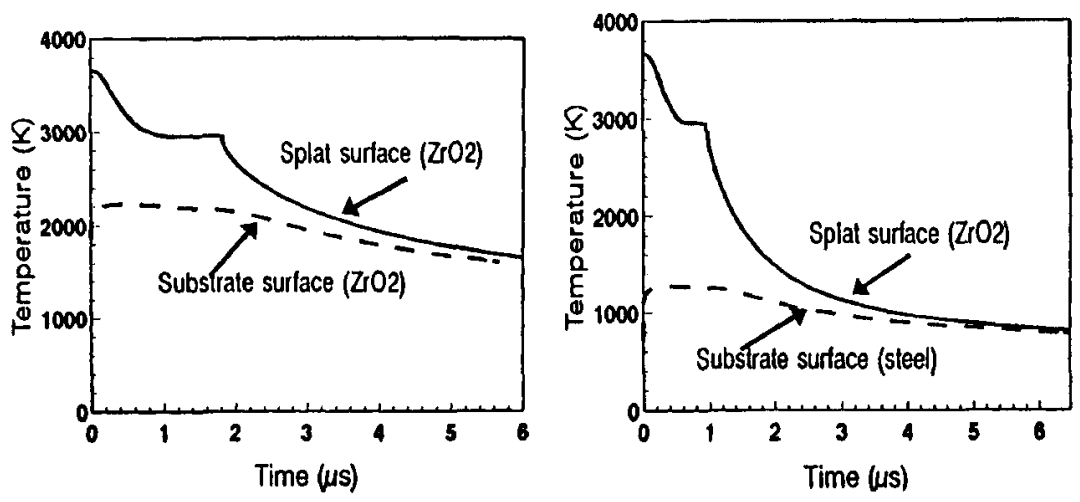

Fig. 11

Time - temperature history of the surface of the splat and the underlying layer : (a) zirconia splat on zirconia surface; (b) zirconia splat on steel layer splat thickness: $1 \mu \mathrm{m}$

Variations in cooling time according to substrate nature are quite minor for thin splats $(<0.5 \mu \mathrm{m})$, but become important as lamella thickness increases up to $1.5 \mu \mathrm{m}$ or more. In the case of a $1-\mu \mathrm{m}$ zirconia splat, the substrate temperature increases up to $1200 \mathrm{~K}$ for a steel substrate, whereas it reaches about $2000 \mathrm{~K}$ for a zirconia substrate as shown in Fig. 11. This appreciable difference lies in the low thermal effusivity of zirconia (about $2800 \mathrm{~J} \mathrm{~m}^{-2} \mathrm{~K}^{-1} \mathrm{~s}^{-0.5}$ ) compared to that of steel (about $9700 \mathrm{~J} \mathrm{~m}^{-2} \mathrm{~K}^{-1} \mathrm{~s}^{-}$ 0.5 ). This low effusivity restrains the diffusion of the heat released by the solidification and cooling of the splat and thus favors the heating of the underlying surface.

\section{CONCLUSION}

In plasma spraying, fluctuations in the rate of electric energy dissipation in the plasma jet, and rapid entrainment of surrounding gas affect the particle trajectories. This result in different trajectories for particles of different sizes and even particles of the same size. Fine particles have difficulty to penetrate in the plasma jet due to their relatively low momentum at the injector exit. They may be entrained by the plasma flow further downstream and thus act on the coating quality. The median trajectory followed by the hottest particles is, in general, at an angle of $3-5^{\circ}$ with the jet centerline.

The rate of evaporation of molten particles in the plasma cone which depends on the temperature they attain, is the highest along that median trajectory. Calculations of the vaporization of iron particles of $5-80 \mu \mathrm{m}$ showed that the mass fraction vaporized amounted to $15 \%$ for a $30-\mu \mathrm{m}$ particle and $1 \%$ for a $60-\mu \mathrm{m}$ particle. For a normal particle distribution, the overall mass fraction vaporized was about $17 \%$. This number was in good agreement with actual measurements of iron particle vaporization carried out by absorption spectroscopy : the summation of iron atoms for particles ranging between 14 and $55 \mu \mathrm{m}$ in a 1-mm slice of the actual plasma jet resulted in a total fraction of vaporized metal of $15 \%$.

The loss of powder at the injection point and the evaporation of particles in the plasma jet are important causes in the lowering of deposit efficiency. Moreover, the particles which have not penetrated in the plasma jet and the vapor cloud around particles may create defects in the coating. Upon impact on the substrate, splat formation depends on the parameters of impinging particles and on the topography, temperature and reactivity of the underlying surface. The cooling rate of the splats is mainly controlled by the quality of the contact with the substrate and the thickness of the splats. It can be as high as $10^{8} \mathrm{~K} / \mathrm{s}$ for the first microseconds following the impact.

\section{REFERENCES}

1. J.F.Coudert, M.P.Planche, P.Fauchais, Plasma Chemistry, Plasma Processing 15, 47 (1995)

2. E.Pfender, J.R. Fincke, R.Spores, Plasma Chemistry, Plasma Processing 11, 529 (1991)

3. J.R.Fincke, W.Swank, Proceedings of the 4th National Thermal Spray Conference, 193 (1991)

4. B.Dussoubs, A.Vardelle, K-I Li, P. Fauchais, Proceedings of the 12th International Symposium on Plasma Chemistry, Minneapolis, 21-25 August (1995)

5. A.Vardelle, J.M.Baronnet, M.Vardelle, P.Fauchais IEEE Transactions on Plasma Science PS-8, 417 (1981)

6. K.G.Shaw, R.German, Proceedings of the 7th.National Thermal Spray Conference, 399 (1994)

7. M. Vardelle, C.Trassy, A.Vardelle, P.Fauchais, Plasma Chemistry and Plasma Processing 11, 185 (1991)

8. N. J. Themelis, Transport and Chemical Rate Phenomena, Gordon and Breach Publishers (1995)

10.G. Chambon, C.Trassy, M.Vardelle, A.Vardelle, P.Fauchais, Journal of High Temperature

Chemical Processes 3, 232-239 (1992)

11.M.Vardelle, A.Vardelle, P.Fauchais, C.Moreau, Meas.Sci.Technol. 5, 205-212 (1994)

12.G.Trapaga, J.Szekely, Metallurgical Transactions B-22, 901-914 (1991)

13.H.Liu, E.J.Lavernia, R.H.Rangel, J.Phys. D : Appl. Phys. 26, 1900-1908 (1993)

(C) 1996 IUPAC, Pure and Applied Chemistry 68, 1093-1099 